\title{
Molecular docking based screening of triterpenoids as potential G-quadruplex stabilizing ligands with anti-cancer activity
}

\author{
Sittichai Sillapapongwarakorn ${ }^{1}$, Somchai Yanarojana ${ }^{1}$, \\ Thithapandha ${ }^{1}$, Jiraporn Ungwitayatorn ${ }^{2}$, Porntip Supavilai ${ }^{1}$ \\ Darawan Pinthong1, \\ Amnuay \\ 1Department of Pharmacology, Faculty of Science, Mahidol University, 272 Rama 6 Road, Bangkok 10400, Thailand; ${ }^{2}$ Department of \\ Pharmaceutical Chemistry, Faculty of Pharmacy, Mahidol University, 447 Sri-Ayudhaya Road, Bangkok 10400, Thailand; Porntip \\ Supavilai - Email: porntip.sup@mahidol.ac.th; Tel: +66 2961 0727; Fax: +66 2961 0727; *Corresponding author
}

Received August 17, 2017; Revised August 30, 2017; Accepted September 2, 2017; Published September 30, 2017

\begin{abstract}
:
Triterpenoids isolated from Ganoderma lucidum (GLTs) exhibit a broad spectrum of anti-cancer properties, including anti-proliferative, anti-metastatic and anti-angiogenic activities. Current research studies revealed the role by GLTs in inducing apoptosis and suppression of telomerase activity of cancer cells with much lower toxicity to healthy cells. Compounds selectively binding and stabilizing G-quadruplex structures could inhibit the telomerase or downregulate the oncogenes and may act as anti-cancer agents. Targeting human telomeric G-quadruplex DNA could be one of the mechanisms by which these GLTs exert anti-cancer activity. In this study, 208 GLTs were screened for ligands with high binding affinity and selectively to stabilize the pG4DNA by using the docking tool AutoDock4. The results showed that ganoderic acid A and ganoderic acid Df exhibit high binding affinity and selectively bind to the lateral groove of pG4DNA. Based on our findings, we suggest that the triterpenoid represents a new class of G-quadruplex groovebinding ligands and thus act as potential anti-cancer agents.
\end{abstract}

Keywords: Ganoderma lucidum, G-quadruplex, molecular docking, triterpenoids, virtual screening

\section{Background:}

Ganoderma lucidum (GL) possesses various pharmacological properties, which are also documented in the ancient reports where GL is praised for its effects on the promotion of health and longevity. It has been known to have numerous pharmacological effects including anti-aging, anti-cancer, anti-diabetic, anti-HIV-1, anti-inflammatory, anti-hypertensive, anti-oxidative, immunemodulating, and radical-scavenging effects [1]. Recently, more than 400 bioactive compounds have been isolated and identified from GL [2]. The main bioactive natural components from GL are triterpenoids, polysaccharides, nucleotides, fatty acids, glycoproteins, sterols, steroids, proteins and trace elements [3]. GL is one attractive source of anti-cancer products, which have been used for centuries as an herbal medicine for the prevention and treatment of cancer and improving immune function. The triterpenoids, structurally highly oxidized lanostanes, have been isolated and characterized with ganoderic acids (GAs) such as ganoderic acid A (GA A), GA D, GA Df, GA T [3-4]. Accumulated data have shown that GAs exhibits a broad spectrum of anticancer properties, including anti-proliferative, anti-metastatic and anti-angiogenic activities [5]. In 2008, Yuen et al. reported that GL extract (GLE) is a potential source of chemopreventive agents for human bladder cancer [6]. Cancer cell growth inhibition induced by GLE is mediated via apoptosis associated with suppression of telomerase activity and oxidative DNA damage [6]. Telomeric DNA contains repetitive DNA sequence (TTAGGG) ${ }_{n}$ forming G-quadruplex (G4) structures; this structure inhibits telomerase activity that is required to maintain telomeres [7]. Therefore, the compounds those selectively binds to and stabilize G4 complex structures could inhibit the telomerase and suppress the gene transcription of oncogenes, which will result in senescence and apoptosis of cancer cells [8].

Several research groups have focused on the structure-based design approaches to develop the potential anti-cancer ligands with the capability to stabilize G4 [9]. Most G4 ligands, such as BRACO19, PIPER, quercetin, RHPS4, telomestatin and TmTyP4, are planar molecules, which comprise a planar p-rich rings structure, allowing them to intercalate into G4 and form a stable conformation [10-15]. Recently, non-planar G4 ligands such as berberine, peimine, peiminine and steroid FG, could stabilize G4 complex through interaction in the lateral groove [16-19]. GLTs 


\section{BIOINFORMATION Discovery at the interface of physical and biological sciences}

\section{Open access}

are non-planar molecules, structurally similar to steroid FG, which may stabilize G4 [17, 18]. GA A was the first GA isolated from GL in 1982 and it shows no cytotoxicity to normal and cancer cells [20, 21]. GA A displays anti-cancer effects, such as anti-invasion, inhibition of NF-kB AP1/uPA, anti-proliferation, inhibition of JAK-STAT3, inhibition of farnesyl protein transferase [22-25]. In this study, GA A was selected to elucidate the capability of GA A to stabilize G4. The parallel stranded DNA quadruplex d- (TTAGGGT) 4 (PDB code 1NP9) were selected for exploiting the interaction of GA A and G4 structure [26]. The aims of the present work were to search for novel GLT ligands with high binding affinity and selectivity for the pG4DNA, which may lead to the discovery of novel natural molecules as lead, compounds having potential anti-cancer activity.

\section{Methodology:}

Preparation of ligand:

The two-dimensional (2D) structures of a total of 208 triterpenoids isolated from Ganoderma lucidum were downloaded from the SCiFinder database. The 3D structures were created with SYBYL 8.0 of NECTEC server. Energy minimization was performed to find the optimum structure with lowest energy. Energy minimization of each structure was achieved by using standard Tripos force field (Powell method and $0.0001 \mathrm{kcal} /$ mole energy gradient convergence criteria). Electrostatic charge was assigned by Gasteiger-Huckel, and iterations number was set to 3,000 rounds.

\section{Molecular docking:}

The structure of the parallel G-quadruplex d-(TTAGGGT)4 containing the human telomeric repeat was retrieved from the protein data bank (PDB code 1NP9); http://www.rcsb.org/pdb) (Gavathiotis and Searle 2003). Dockings of GLTs to unbound pG4DNA were carried out using AutoDock 4 with AutoDockTools 1.5.6rc3 (ADT) as described by Li J et al. [18]. G4 structures were prepared for docking using Sybyl 8 software (Certara Inc. Princeton, USA) and ADT. The Gasteiger atomic charges and Kollman united atom partial charges were assigned for the GLTs and G4, respectively. Grid maps were set at four grooves and two terminal places for G4 and the grid boxes were centered at G4. The size of grid box was $60 \times 60 \times 60$ in three dimensions. The grid was set to be sufficiently large to cover significant portions of the active sites. Lamarckian genetic search algorithm was employed. Maximum number of energy evaluation was 2, 500, 000 per run and population size was set at 150. All other parameters were set to default values. Two hundred fifty independent docking runs were performed. Results were divided into groups using the clustering module in ADT according to the 2.0 root-mean-square deviation (RMSD) criteria. Besides RMSD clustering, the binding free energies were evaluated for the binding conformations of ligand by using ADT, and the low-energy conformations were selected from the largest cluster [18].

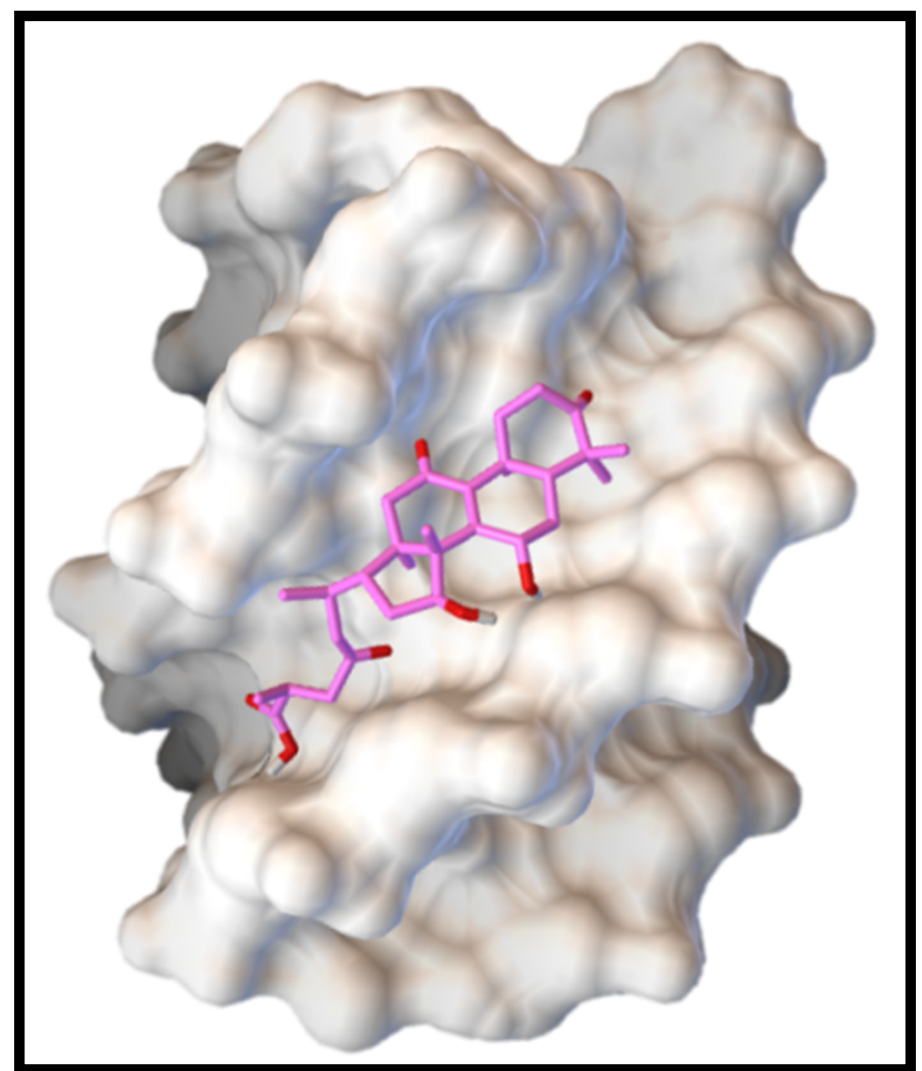

Figure 1: Predicted binding mode and the possible binding site of GA A with pG4DNA. GA A, represented in pink sticks, was able to enter and filled the binding groove of pG4DNA. Abbreviations: pG4DNA, parallel G-quadruplex DNA; GA, ganoderic acid.

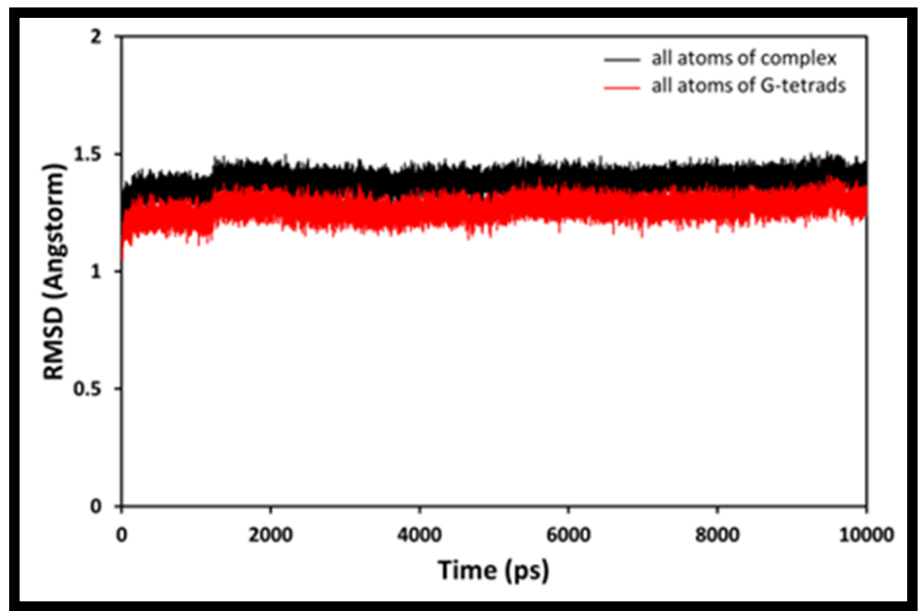

Figure 2: Structural drift observed during the MD simulation. RMSD plot showing the stability of the model during the MD run. RMSD values calculated for all atoms of GA A-pG4DNA complex (red) and backbone only atoms of pG4DNA (black) were plotted. 


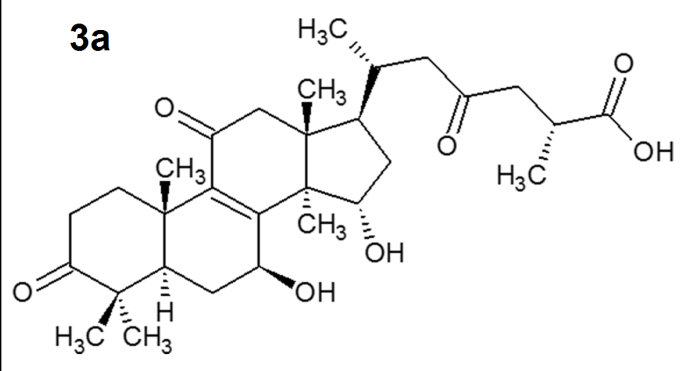
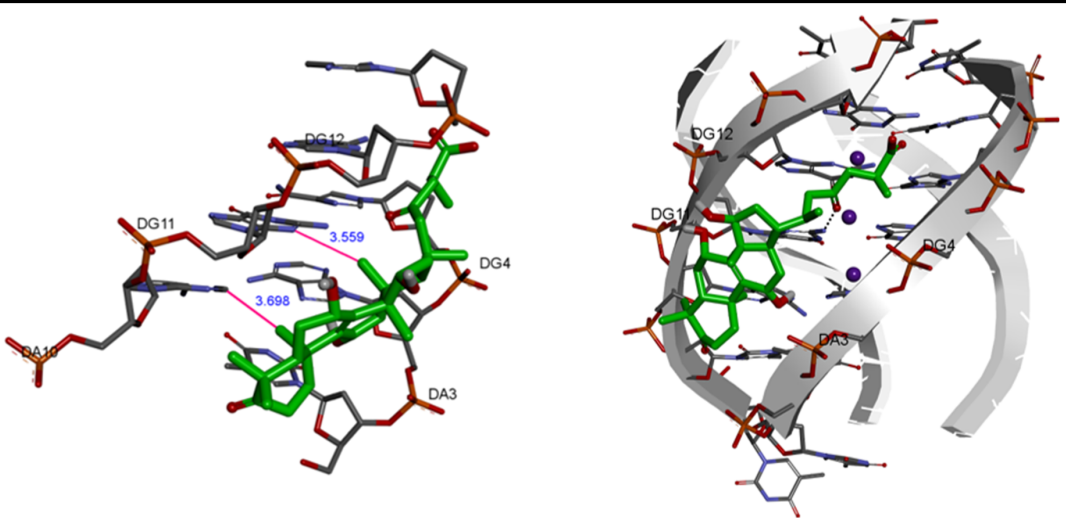

3b<smiles></smiles>

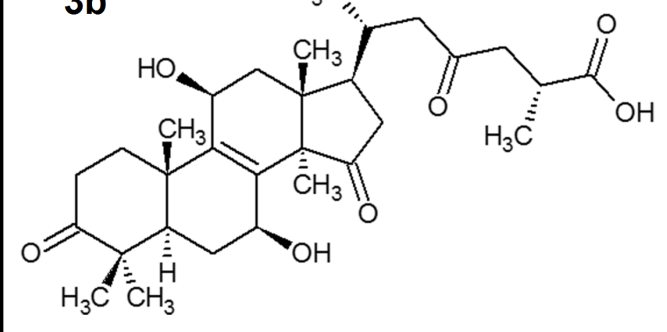
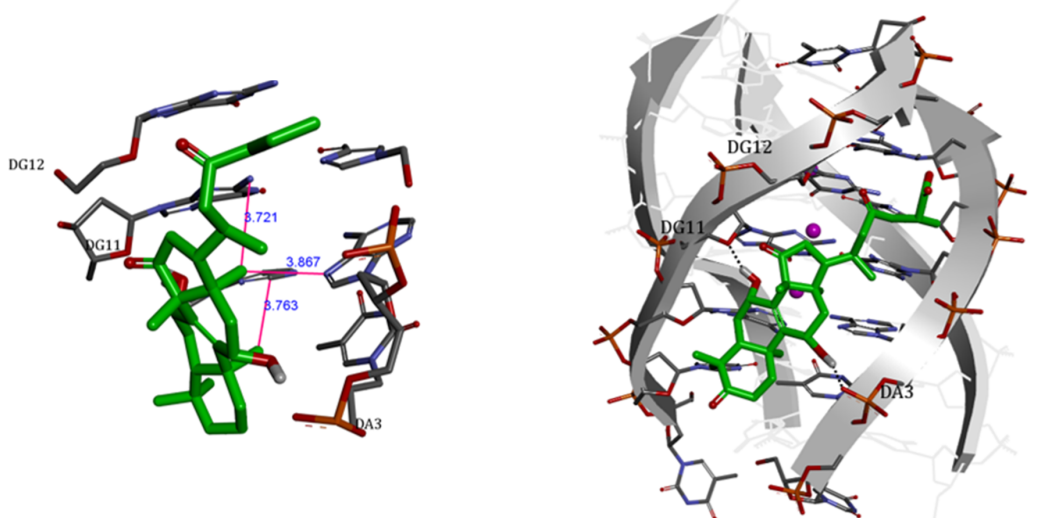

Figure 3: Binding poses of the (a) GA (Ganoderic acid) A and (b) GA (Ganoderic acid) Df in the pG4DNA-binding site. The figures shown are the predicted interactions formed by the ganoderic acid A in the active site. The compounds are represented in green sticks. The purple balls are $\mathrm{K}^{+}$. The pG4DNA structure is shown as a gray ribbon diagram with exception to the activation loop containing the DA-motif and DG-motif, which is shown in red sticks. The black dash lines represent hydrogen bonds, and purple lines denote hydrophobic interactions.

Abbreviations: DA3, adenine base position 3 of G4DNA; DG11, guanine base position 11 of pG4DNA; pG4DNA, parallel Gquadruplex DNA; GA, ganoderic acid.

\section{Molecular dynamics:}

Molecular dynamic (MD) simulations for the GLTs-G4 systems were carried out with the sander module of AMBER 12.0 program package as described by Li J et al. [18]. The PARM 99 parameters and General Amber Force Field (GAFF) parameters were set for G4 and GLTs, respectively. Partial-atomic charges for the triterpenoids were derived using Gaussian 03 with the HF/6$31 \mathrm{G}$ (d) basis set followed by RESP calculation. Internal $\mathrm{K}^{+}$ion was added into the channels of the cavities between consecutive guanine tetrads. Then, the TIP3P water model was chosen and extended to a distance of $10 \AA$ from any solute atom. Counter K+ ion neutralized the systems.

Firstly, a 1000-step minimization was carried out with the solute molecules fixed, and the equilibration was continued by 20 ps of PME dynamics with the same restriction. Subsequently, five rounds of 1,000-step minimization followed this equilibration with solute restraints from 20 to $0 \mathrm{kcal} / \mathrm{mol} \AA^{-2}$ reduced by $5 \mathrm{kcal}$ $\mathrm{kcal} / \mathrm{mol} \AA^{-2}$ in the course of each round. Then, the system was heated from 0 to $300 \mathrm{~K}$ with the rate of $50 \mathrm{~K}$ for every 5 ps of MD run, and another 100 ps MD simulation was continued to ISSN 0973-2063 (online) 0973-8894 (print) equilibrate the system. After the minimization and equilibration, MD simulations were run under NPT condition at $300 \mathrm{~K}$. During the MD simulations, SHAKE was used for constraining hydrogen atoms and a 9 cutoff was applied to non-bonded interaction. Simulation time step was set at $2 \mathrm{fs}$ and the translational center of mass motion was removed every 10 ps [18].

\section{Results and Discussion:}

\section{Molecular docking and MD stimulations:}

GA A, which exhibits anti-cancer effects and shows no cytotoxicity to normal and cancer cells [20, 21], was selected to evaluate the ability to stabilize pG4DNA. The docking result of GA A in pG4DNA binding site is shown in Figure 1. The lowest energy docked conformation of the most populated cluster (the largest cluster) was selected and taken into account for study the binding against pG4DNA. The estimated inhibition constant (Ki) and estimated free binding energy $(\triangle G)$ of GA A which stabilized the pG4DNA are shown in Table 1. The lowest binding free energy conformation of GA A binding in pG4DNA was selected for further MD stimulation. 
Table 1: Docking summary of pG4DNA (1NP9) with 208 currently known GLTs.

\begin{tabular}{|c|c|c|c|c|}
\hline Compound No. & Compounds & CAS No. & Estimate $\triangle \mathrm{G}(\mathrm{kcal} / \mathrm{mol})$ & Estimate average $\mathrm{Ki}(\mathrm{nM})$ \\
\hline 1 & GA A & $81907-62-2$ & -8.76 & 376.92 \\
\hline 2 & GA AM1 & 149507-55-1 & -9.79 & 66.95 \\
\hline 3 & GA AP & $120462-50-2$ & -9.44 & 121.01 \\
\hline 4 & GA AP2 & 1082416-00-9 & -8.09 & 1,170 \\
\hline 5 & GA AP3 & $1082416-03-2$ & -9.57 & 97.06 \\
\hline 6 & GA B & 81907-61-1 & -8.46 & 633.32 \\
\hline 7 & GA B8 & 105817-07-0 & -9.34 & 141.46 \\
\hline 8 & GA B9 & - & -8.95 & 274.61 \\
\hline 9 & GA C2 & $103773-62-2$ & -8.93 & 282.57 \\
\hline 10 & GA C5 & $673460-24-7$ & -9.41 & 126.81 \\
\hline 11 & GA C6 & $105742-76-5$ & -7.51 & 3,130 \\
\hline 12 & GA D & $108340-60-9$ & -8.89 & 305.82 \\
\hline 13 & GA D1 & - & -9 & 254.25 \\
\hline 14 & GA D2 & $97653-94-6$ & -9.23 & 171.1 \\
\hline 15 & GA Df & $1352033-73-8$ & -10.72 & 13.97 \\
\hline 16 & GA DM & $173075-45-1$ & -9 & 254.56 \\
\hline 17 & GA E & $98665-14-6$ & -9.96 & 50.39 \\
\hline 18 & GA F & $98665-15-7$ & -9.07 & 226.16 \\
\hline 19 & GA G & $98665-22-6$ & -8.54 & 545.63 \\
\hline 20 & GA GS-1 & $1206781-64-7$ & -8.67 & 444.3 \\
\hline 21 & GA GS-2 & $1206781-65-8$ & -8.76 & 379.65 \\
\hline 22 & GA GS-3 & $1206781-66-9$ & -7.86 & 1,740 \\
\hline 23 & GA H & $98665-19-1$ & -7.05 & 6,770 \\
\hline 24 & GA I & $98665-20-4$ & -9.16 & 192.28 \\
\hline 25 & GA J & $100440-26-4$ & -9.48 & 112.35 \\
\hline 26 & GA Ja & $112430-67-8$ & -8.87 & 314.29 \\
\hline 27 & GA Jb & $112430-68-9$ & -8.95 & 273.93 \\
\hline 28 & GA K & $104700-95-0$ & -8.4 & 696.77 \\
\hline 29 & GA L & $102607-24-9$ & -9.54 & 101.48 \\
\hline 30 & GA LM2 & $508182-41-0$ & -8.39 & 713.51 \\
\hline 31 & GA M & $110311-47-2$ & -9.36 & 138.4 \\
\hline 32 & GA Ma & $108026-89-7$ & -6.17 & 30,240 \\
\hline 33 & $\mathrm{GA} \mathrm{Mb}$ & $108026-90-0$ & -6.89 & 8,900 \\
\hline 34 & GA Mc & 108026-91-1 & -7.07 & 6,610 \\
\hline 35 & GA Md & $108026-92-2$ & -6.53 & 16,330 \\
\hline 36 & $\mathrm{GA} \mathrm{Me}$ & $108026-93-3$ & -7.09 & 6,350 \\
\hline 37 & GA Mf & $108026-94-4$ & -8.77 & 369.98 \\
\hline 38 & GA Mg & $110042-11-0$ & -7.18 & 5,430 \\
\hline 39 & GA Mh & $110024-17-4$ & -7.04 & 6,900 \\
\hline 40 & GA Mi & $110024-16-3$ & -8.03 & 1,290 \\
\hline 41 & GA Mj & $110024-15-2$ & -7.19 & 5,360 \\
\hline 42 & GA Mk & 110024-14-1 & -7.97 & 1,440 \\
\hline 43 & GA N & $110241-19-5$ & -9.2 & 181.04 \\
\hline 44 & GA O & $110241-21-9$ & -10.12 & 38.28 \\
\hline 45 & GA P & $112667-14-8$ & -7.3 & 4,450 \\
\hline 46 & GA R & 103963-39-9 & -7.46 & 3,410 \\
\hline 47 & GA S & $104759-35-5$ & -8.78 & 369.21 \\
\hline 48 & GA SZ & $865543-37-9$ & -8.99 & 255.68 \\
\hline 49 & GA T & 103992-91-2 & -5.73 & 62,870 \\
\hline 50 & GA TN & $112430-64-5$ & -7.67 & 2,400 \\
\hline 51 & GA TR & $862893-75-2$ & -9.14 & 199.55 \\
\hline 52 & GA TR1 & $1225286-05-4$ & -9.32 & 148.21 \\
\hline 53 & GA T-Q & $112430-66-7$ & -8.1 & 1,160 \\
\hline 54 & GA U & $86377-51-7$ & -8 & 1,360 \\
\hline 55 & GA V & $86377-50-6$ & -7.29 & 4,570 \\
\hline
\end{tabular}

ISSN 0973-2063 (online) 0973-8894 (print) 


\begin{tabular}{|c|c|c|c|c|}
\hline 56 & GA V1 & 150033-91-3 & -8.55 & 542.99 \\
\hline 57 & GA W & $86377-49-3$ & -6.75 & 11,270 \\
\hline 58 & GA X & $86377-53-9$ & -7.97 & 1,440 \\
\hline 59 & 3 - $\beta$-hydroxy GA X & - & -7.86 & 1,730 \\
\hline 60 & GA Y & $86377-52-8$ & -8.49 & 602.92 \\
\hline 61 & GA Z & 86420-19-1 & -8.18 & 1,020 \\
\hline 62 & GA $\alpha$ & $220181-81-7$ & -7.65 & 2,460 \\
\hline 63 & GA $\beta$ & 217476-76-1 & -8.46 & 631.78 \\
\hline 64 & GA $\gamma$ & 294674-00-3 & -8.83 & 338.2 \\
\hline 65 & GA $\delta$ & 294674-02-5 & -8.96 & 272.38 \\
\hline 66 & GA $\varepsilon$ & 294674-05-8 & -8.83 & 338.29 \\
\hline 67 & $\mathrm{GA} \zeta$ & 294674-09-2 & -10.16 & 35.86 \\
\hline 68 & GA $\eta$ & 294674-12-7 & -8.2 & 979.27 \\
\hline 69 & GA $\theta$ & 294674-15-0 & -8.77 & 370.79 \\
\hline 70 & Ganodermic acid S & $112430-63-4$ & -7.7 & 2,290 \\
\hline 71 & Ganodermic acid T-O & $112430-65-6$ & -8.59 & 507.25 \\
\hline 72 & Ganoderenic acid A & $100665-40-5$ & -8.69 & 426.02 \\
\hline 73 & Ganoderenic acid A & - & -9.49 & 111.51 \\
\hline 74 & Ganoderenic acid B & $100665-41-6$ & -6.89 & 8,860 \\
\hline 75 & Ganoderenic acid C & $100665-42-7$ & -6.89 & 8,910 \\
\hline 76 & Ganoderenic acid D & $100665-43-8$ & -7.17 & 5,590 \\
\hline 77 & Ganoderenic acid E & $110241-23-1$ & -6.8 & 10,380 \\
\hline 78 & Ganoderenic acid F & $120462-47-7$ & -7.63 & 2,550 \\
\hline 79 & Ganoderenic acid G & $120481-73-4$ & -7.71 & 2,230 \\
\hline 80 & Ganoderenic acid $\mathrm{H}$ & $120462-48-8$ & -7.54 & 2,970 \\
\hline 81 & Ganoderenic acid I & $120462-49-9$ & -7.35 & 4,120 \\
\hline 82 & Ganoderenic acid K & $942950-94-9$ & -7.72 & 2,180 \\
\hline 83 & Methyl GA A & $81907-63-3$ & -9.1 & 214.48 \\
\hline 84 & Methyl GA AP & $120462-52-4$ & -8.94 & 280.93 \\
\hline 85 & Methyl GA B & $81907-65-5$ & -7.8 & 1,910 \\
\hline 86 & Methyl GA D & $97210-12-3$ & -9.07 & 225.56 \\
\hline 87 & Methyl GA Df & $1351348-00-9$ & -10.28 & 29.18 \\
\hline 88 & Methyl GA DM & - & -8.51 & 573.98 \\
\hline 89 & Methyl GA E & $98718-43-5$ & -9.58 & 95.72 \\
\hline 90 & Methyl GA F & $98665-08-8$ & -9.97 & 49.26 \\
\hline 91 & Methyl GA G & $98665-23-7$ & -8.56 & 531.94 \\
\hline 92 & Methyl GA H & $98665-11-3$ & -7.1 & 6,220 \\
\hline 93 & Methyl GA I & $98683-73-9$ & -8.15 & 573.68 \\
\hline 94 & Methyl GA K & $110414-79-4$ & -9.17 & 189.33 \\
\hline 95 & Methyl GA K2003 & $105742-77-6$ & -8.86 & 318.33 \\
\hline 96 & Methyl Ganoderenic acid H & $120462-54-6$ & -7.25 & 4,860 \\
\hline 97 & Methyl Ganoderenic acid I & $120462-53-5$ & -7.18 & 5,480 \\
\hline 98 & Ethyl GA F & $1245946-63-7$ & -10.16 & 35.97 \\
\hline 99 & Propyl GA F & - & -10.27 & 29.47 \\
\hline 100 & i-Propyl GA F & - & -10.14 & 36.96 \\
\hline 101 & Butyl GA F & - & -10.09 & 40.43 \\
\hline 102 & i-Butyl GA F & - & -10.34 & 26.23 \\
\hline 103 & s-Butyl GA F & - & -9.27 & 75.21 \\
\hline 104 & t-Butyl GA F & - & -10.17 & 35.18 \\
\hline 105 & Butyl GA A & 1207106-19-1 & -9.41 & 126.76 \\
\hline 106 & Butyl GA B & $1207106-20-4$ & -8.46 & 634.33 \\
\hline 107 & Tri-OAc Ganodermatriol & $1028449-54-8$ & -9.21 & 176.11 \\
\hline 108 & Ganodermatriol M & - & -9.31 & 150.67 \\
\hline 109 & 3-OAc GA B & - & -8.47 & 619.16 \\
\hline 110 & 12-hydroxy GA C2 & $942936-52-9$ & -8.99 & 256.31 \\
\hline 111 & 12- $\alpha$-hydroxy GA D & - & -9.94 & 51.72 \\
\hline 112 & 12- $\alpha-\mathrm{OAc}$ GA D & $942936-55-2$ & -9.84 & 61.51 \\
\hline
\end{tabular}

ISSN 0973-2063 (online) 0973-8894 (print) 


\begin{tabular}{|c|c|c|c|c|}
\hline 113 & 15-OAc Ganolucidic acid E & 1309931-94-9 & -7.64 & 2,500 \\
\hline 114 & 12-hydroxy GA F & - & -10.29 & 28.49 \\
\hline 115 & 20-hydroxy GA G & $400604-12-8$ & -9.76 & 70.07 \\
\hline 116 & 3-OAc GA K & - & -8.79 & 358.12 \\
\hline 117 & 3-OAc GA H & $942936-56-3$ & -7.07 & 6,570 \\
\hline 118 & 7-methyl GA O & $112667-15-9$ & -7.3 & 4,490 \\
\hline 119 & 7-ethyl GA O & - & -6.32 & 23,130 \\
\hline 120 & 15-hydroxy GA S & - & -9.07 & 223.84 \\
\hline 121 & 23-hydroxy GA S & $1225286-07-6$ & -8.7 & 422.67 \\
\hline 122 & 7-carbonyl GA Z & - & -8.36 & 742.06 \\
\hline 123 & 7-carbonyl methyl GA Z & - & -7.83 & 1,820 \\
\hline 124 & 2-OAc methyl GA A & $81907-64-4$ & -7.53 & 3,030 \\
\hline 125 & 7-hydroxy methyl GA AP & $120481-75-6$ & -9.1 & 214.81 \\
\hline 126 & Me89GA J & 400604-11-7 & -9.65 & 84.48 \\
\hline 127 & Lucidenic acid A & 95311-94-7 & -8.31 & 807.65 \\
\hline 128 & Lucidenic acid B & $95311-95-8$ & -8.2 & 981.84 \\
\hline 129 & Lucidenic acid C & $95311-96-9$ & -7.51 & 3,130 \\
\hline 130 & Lucidenic acid D & $98665-16-8$ & -9.1 & 212.66 \\
\hline 131 & Lucidenic acid D1 & 97653-95-7 & -9.01 & 250.71 \\
\hline 132 & Lucidenic acid E & $98665-17-9$ & -8.57 & 523.96 \\
\hline 133 & Lucidenic acid E1 & $97673-89-7$ & -8.24 & 911.36 \\
\hline 134 & Lucidenic acid F & $98665-18-0$ & -9.05 & 231.93 \\
\hline 135 & Lucidenic acid $G$ & $102607-21-6$ & -8.09 & 1,180 \\
\hline 136 & Lucidenic acid $\mathrm{H}$ & $110241-25-3$ & -7.71 & 2,230 \\
\hline 137 & Lucidenic acid I & $110241-27-5$ & -9.54 & 101.02 \\
\hline 138 & Lucidenic acid J & $110241-29-7$ & -8.7 & 422.78 \\
\hline 139 & Lucidenic acid $\mathrm{K}$ & $110241-31-1$ & -8.86 & 318.4 \\
\hline 140 & Lucidenic acid L & $110267-45-3$ & -8.46 & 633.21 \\
\hline 141 & Lucidenic acid M & $110241-33-3$ & -8 & 1,370 \\
\hline 142 & Lucidenic acid $\mathrm{O}$ & $250643-33-5$ & -7.49 & 3,250 \\
\hline 143 & Lucidenic acid P & $648430-31-3$ & -7.32 & 4,310 \\
\hline 144 & Lucidenic acid SP1 & $364622-33-3$ & -7.77 & 2,030 \\
\hline 145 & 20deHLA A & $852396-69-7$ & -7.57 & 2,800 \\
\hline 146 & 20deHLA N & $1206781-67-0$ & -7.39 & 3,860 \\
\hline 147 & 20OHLA A & $1206781-68-1$ & -8.25 & 894.69 \\
\hline 148 & 20OHLA D2 & 852936-71-1 & -8.88 & 310.28 \\
\hline 149 & 20OHLA E2 & $852567-75-0$ & -8.71 & 411.51 \\
\hline 150 & 20OHLA F & $852567-72-7$ & -9.16 & 192.27 \\
\hline 151 & 20OHLA N & $852567-78-3$ & -8.16 & 1,040 \\
\hline 152 & 20OHLA P & $852567-80-7$ & -7.74 & 2,130 \\
\hline 153 & LAlactone & 250643-34-6 & -6.91 & 8,640 \\
\hline 154 & Lucideraldehyde A & 420781-84-6 & -7.79 & 1,960 \\
\hline 155 & Lucideraldehyde B & 480439-84-7 & -8.41 & 687.6 \\
\hline 156 & Lucideraldehyde C & $252351-96-5$ & -8.45 & 644.96 \\
\hline 157 & Lucideraldehyde D & $873061-78-0$ & -8.29 & 837.72 \\
\hline 158 & Ganolucidic acid A & $98665-21-5$ & -9.77 & 69.51 \\
\hline 159 & Ganolucidic acid B & $98683-75-1$ & -9.42 & 123.9 \\
\hline 160 & Ganolucidic acid C & $100440-27-5$ & -8.77 & 370.2 \\
\hline 161 & Ganolucidic acid D & $102607-22-7$ & -9.16 & 193.85 \\
\hline 162 & Ganolucidic acid E & $114567-50-9$ & -9.05 & 234.28 \\
\hline 163 & Ganosporic acid A & $135357-25-4$ & -9.66 & 83.46 \\
\hline 164 & trideOAcGA_T & 116763-90-7 & -8.52 & 571.65 \\
\hline 165 & MeLA A & $105742-79-8$ & -7.24 & 4,910 \\
\hline 166 & MeLA C & $98094-88-3$ & -8.17 & 1,020 \\
\hline 167 & MeLA D2 & $98665-09-9$ & -8.88 & 311.87 \\
\hline 168 & MeLA E2 & $98665-12-4$ & -8.4 & 692.6 \\
\hline 169 & MeLA F & $98665-10-2$ & -8.92 & 291.39 \\
\hline
\end{tabular}

ISSN 0973-2063 (online) 0973-8894 (print) 


\begin{tabular}{|c|c|c|c|c|}
\hline 170 & MeLA L & $110267-46-4$ & -8.34 & 767.46 \\
\hline 171 & MeLA N & $1276655-49-2$ & -7.62 & 2,620 \\
\hline 172 & MeLA P & $647856-35-7$ & -7.11 & 6,120 \\
\hline 173 & MeLA Q & $648430-32-4$ & -8.03 & 1,310 \\
\hline 174 & MeGlA A & $98665-13-5$ & -9.48 & 111.8 \\
\hline 175 & MeGlA B & $98683-74-0$ & -9.32 & 146.31 \\
\hline 176 & MeGlA D & $102607-26-1$ & -9.04 & 234.95 \\
\hline 177 & Me20deHLA A & 852936-70-0 & -7.19 & 5,340 \\
\hline 178 & BuLA A & $1207106-22-6$ & -8.36 & 740.68 \\
\hline 179 & BuLA B & $1314143-37-7$ & -8.23 & 928.49 \\
\hline 180 & BuLA N & $1207106-21-5$ & -7.35 & 4,090 \\
\hline 181 & Ganoderal A & 106518-61-0 & -7.74 & 2,130 \\
\hline 182 & Ganoderal B & $106518-62-1$ & -9.56 & 97.8 \\
\hline 183 & Ganoderal F & $114567-47-4$ & -9.17 & 190.81 \\
\hline 184 & Ganodermadiol & 104700-96-1 & -7.77 & 2,000 \\
\hline 185 & Ganodermatriol & $105300-28-5$ & -8.06 & 1,230 \\
\hline 186 & Ganodermanonol & $104700-97-2$ & -8.6 & 494.44 \\
\hline 187 & GAdiol 2006 & $107900-76-5$ & -8.66 & 452.08 \\
\hline 188 & GAtriol & $106518-63-2$ & -8.47 & 622.13 \\
\hline 189 & 89epoxyGA C & - & -9.08 & 221.51 \\
\hline 190 & $89 \mathrm{GAC}$ & - & -8.73 & 401.72 \\
\hline 191 & 89GA J & 400604-10-6 & -8.89 & 303.47 \\
\hline 192 & Ganolactone A & $173268-82-1$ & -7.86 & 1,740 \\
\hline 193 & Ganolactone B & $1028449-53-7$ & -7.72 & 2,210 \\
\hline 194 & Gsl A & $138008-04-5$ & -8.44 & 650.14 \\
\hline 195 & Gsl B & $138008-05-6$ & -7.37 & 3,960 \\
\hline 196 & Furano GA & $120481-74-5$ & -7.12 & 6,060 \\
\hline 197 & EpGOH A & $114020-56-3$ & -8.04 & 1,280 \\
\hline 198 & EpGOH B & $114020-57-4$ & -8.36 & 739.99 \\
\hline 199 & EpGOH C & $114020-58-5$ & -8.25 & 896.93 \\
\hline 200 & GaldTR & $1225286-06-5$ & -8.75 & 385.99 \\
\hline 201 & GamdT & $1341220-87-8$ & -7.25 & 4,860 \\
\hline 202 & Ganoderal A & $104700-98-3$ & -8.4 & 694.04 \\
\hline 203 & Ganoderal B & $114020-55-2$ & -7.76 & 2,070 \\
\hline 204 & Ganoderon B & $252351-95-4$ & -7.42 & 3,620 \\
\hline 205 & Ganoderone A & 873061-79-1 & -8.26 & 882.3 \\
\hline 206 & Ganoderone C & $873061-80-4$ & -8.47 & 615.75 \\
\hline 207 & Lucidumol A & $217476-73-8$ & -9.08 & 222.02 \\
\hline 208 & Lucidumol B & $107900-79-8$ & -8.8 & 356.42 \\
\hline
\end{tabular}

Abbreviations: $\triangle \mathrm{G}$, free binding energy; Ki, inhibition constant; GA, ganoderic acid; pG4DNA, parallel G-quadruplex DNA; GLTs, triterpenoids isolated from Ganoderma lucidum.

Table 2: MM/GBSA binding energies of GA A and GA Df to pG4DNA and residues involved in the G4-ligand interactions.

\begin{tabular}{lllllll}
\hline Triterpenoids & $\Delta_{\mathrm{VDW}^{\mathrm{a}}}(\mathbf{k c a l} / \mathbf{m o l})$ & $\Delta_{\mathrm{SUR}^{\mathrm{b}}} \mathbf{( \mathbf { k c a l } / \mathbf { m o l } )}$ & $\Delta_{\mathrm{GBELE}^{\mathrm{c}}}(\mathbf{k c a l} / \mathbf{m o l})$ & $\Delta_{\mathrm{TOT}^{\mathrm{d}}}(\mathbf{k c a l} / \mathbf{m o l})$ & \multicolumn{2}{l}{ Residues involved in } \\
H-bonding & H-bond length $(\AA)$ \\
\hline GA A & $-49.47 \pm 1.57$ & $-3.93 \pm 0.04$ & $29.94 \pm 1.61$ & $-23.46 \pm 1.70$ & DG 11 & 2.21 \\
& & & & & DG 11 & 2.12 \\
GA Df & $-44.76 \pm 2.17$ & $-3.84 \pm 0.04$ & $35.28 \pm 2.51$ & $-13.32 \pm 2.21$ & DA 3 & 2.74 \\
\hline
\end{tabular}

${ }^{\mathrm{a}} \Delta_{\mathrm{VDw}}$ is the change in van der Waals energy in the gas phase upon complex formation. ${ }^{\mathrm{b}} \Delta_{\text {sur }}$ is the change in energy due to the change in surface area upon complex formation. ${ }^{c} \Delta_{\text {GBELE }}$ is the change in GB reaction field energy + gas phase electrostatic energy upon complex formation. $\mathrm{d} \Delta \Delta_{\mathrm{TOT}}=\Delta \mathrm{VDW}+\Delta \mathrm{SUR}+\Delta_{\mathrm{GBELE}}$ is the change in potential energy in water upon complex formation.

Abbreviations: MM-GBSA, molecular mechanics/ generalized Born surface area; DA 3, adenine base of G-quadruplex DNA at position 3; DG 11, guanine base of G-quadruplex DNA at position 11; GA, ganoderic acid.

Measuring the RMSD over the course of the MD simulation assessed the conformational stability of the GA A-pG4DNA complex. The overall RMSD for all atoms of GA A-pG4DNA complex (red) and backbone-only atoms of pG4DNA (black) are ISSN 0973-2063 (online) 0973-8894 (print)

Bioinformation 13(9): 284-292 (2017) illustrated in Figure 2. There were very few differences in the RMSD values observed between an all atom of GA A-pG4DNA complex and backbone-only model for the G-quartets. The stability of GA A-pG4DNA complex using RSMD calculations 


\section{Open access}

revealed that the binding of GA A was stable. Figure 3 (a) depicts GA A binding in the groove of pG4DNA through hydrogen bond and van der Waals interactions. Thus, GA A could be a potential novel natural molecule that can stabilize pG4DNA. Recently, more than 200 GLTs have been isolated and identified from GL [2]. Therefore, in silico screening of GLTs was performed to search for the GLTs with high binding affinity and selectivity for the pG4DNA.

Virtual screening of GLTs as potential G4 stabilizing ligands : In this study, 208 triterpenoids isolated from GL were screened for the ligand with high binding affinity and selectivity for the pG4DNA. GLTs were docked to the pG4DNA. The structures, estimated $\mathrm{Ki}$ and estimated $\triangle \mathrm{G}$ of GLTs, which stabilized the pG4DNA, are shown in Table 1 . The results indicated that 131 GLTs interact with the pG4DNA with high affinity $(\mathrm{Ki}<1 \mathrm{mM})$. GA Df was the most potent GAs to stabilize the pG4DNA with Ki $=13.97 \mathrm{nM}$

\section{MD stimulations:}

MD stimulations were performed on GA A and GA Df with pG4DNA to explore the binding poses in depth. Molecular Mechanics and Generalized Born Surface Area (MM/GBSA) were determined for the best ranking conformation molecule on the solvation forces involved in the stabilization of GA-pG4DNA complex. The estimated $\mathrm{rG}$, estimated $\mathrm{Ki}$ and target residues involved in the hydrogen bonding of the best-docked poses are given in Table 2. The pG4DNA (1NP9: containing the human telomeric repeat) consists of four equivalent grooves [26]. The results showed that GA A interacts with pG4DNA in the groove through hydrogen bond and van der Waals interactions. One hydrogen bond was formed by side chain carbonyl group of GA $A$ and guanine base position 11 of lateral groove of pG4DNA (DG11) with hydrogen bond length of $2.21 \AA$ A. Two methyl groups (C18 and C19) were pointed into the groove and bound with guanine bases by hydrophobic and van der Waals interactions. The distance between methyl group (C18) and carbon atom of DG11 was $3.7 \AA$, and the distance between methyl group (C19) and nitrogen atom of DA10 was $3.6 \AA$ (Figure 3a and Table 2). GA Df stabilized pG4DNA with 3 van der Waals interactions and 2 hydrogen bonds with pG4DNA at DG 11 and adenine base position 3 (DA3) with hydrogen bond length of $2.12 \AA$ and 2.74 $\AA$, respectively (Figure $3 \mathbf{b}$ and Table 2 ). Hydroxyl group of ring B of GA Df formed hydrogen bond with DG 11 and hydroxyl group of ring $\mathrm{C}$ formed $\mathrm{H}$-bond with DA 3. Two methyl groups (C18 and C19) were pointed into the groove and bound with guanine base by hydrophobic and van der Waals interactions. The distance between methyl group (C18) and nitrogen atom of DG11 was $3.7 \AA$ and the distance of this methyl group and nitrogen atom of DA3 was $3.9 \AA$. The distance between methyl group (C19) and carbon atom of DA10 was $3.8 \AA$. The result also provided new insight into the design of G4 groove-targeted agents.

Furthermore, Table 2 shows the MM/GBSA binding energy calculation of GA A and GA Df to pG4DNA. GA A displayed a lower total binding energy $(-23.46 \pm 1.70 \mathrm{kcal} / \mathrm{mol})$ than GA Df $(-$ $13.32 \pm 2.21 \mathrm{kcal} / \mathrm{mol})$. However, in docking experiment, GA Df ISSN 0973-2063 (online) 0973-8894 (print) was about 30 times more active than GA A (Table 1). As for the results from $\mathrm{MD}$, the interaction of GAs with pG4DNA was in solution which mimicked the physiological condition, the total binding energy of GA A was approximately 2 times better than GA Df. Further studies are required for clarify these results.

The obtained results are in agreement with the published nonplanar G4 ligands that GLTs stabilized G4 through the groove binding [16-19]. The GLTs interacted with the pG4DNA and enhanced G4 stabilization through hydrogen bonds and van der Waals interactions. At physiological condition GA A, noncytotoxic GLTS [20, 21], might be potential lead compounds for the development of new telomerase inhibitors. Thus, GA A may serve as the starting point for the design of a new class of highly selective groove binding of pG4DNA with anti-cancer effect.

\section{Conclusion:}

In conclusion, the first virtual screening of GLTs as potential G4 stabilizing ligands was presented. Binding poses and binding energies for GLT-pG4DNA complexes were calculated using molecular docking and molecular dynamics. The results indicated that GLTs significantly stabilized the pG4DNA through interaction with the lateral groove of G4 by hydrogen bonds and van der Waals forces. GA A and GA Df exhibit high binding affinity and selectivity for lateral groove of pG4DNA with theoretical binding efficiency in nanomolar range. The triterpenoid represents a new class of highly selective groove-binding molecules. Thus, GLTs exert their novel anti-cancer mechanism by stabilizing the pG4DNA through the groove binding.

\section{Acknowledgements:}

This research work was supported by grants from the National Research Council of Thailand and Mahidol University. Science Achievement Scholarship of Thailand supported Sittichai Sillapapongwarakorn.

\section{Competing interests:}

The authors declare that they have no conflict of interests.

\section{References:}

[1] Boh B et al. Biotechnol Annu Rev. 2007 13:265 [PMID; 17875480].

[2] Shao Y et al. Molecules. 2016 21:678 [PMID: 27213329].

[3] Kao CHJ et al. Funct Food Health Dis. 2013 3:48

[4] Fatmawati S et al. Fitoterapia. 2010 81:1033 [PMID: 20603199].

[5] Wu GS et al. Expert Opin Investig Drugs. 2013 22:981 [PMID: 23790022].

[6] Yuen JW et al. Nutr Cancer. 2008 60:109 [PMID: 18444142].

[7] Read M et al. Proc Natl Acad Sci USA. 2001 98:4844 [PMID: 11309493]

[8] Balasubramanian S et al. Nat Rev Drug Discov. 2011 10:261 [PMID: 21455236].

[9] Artese A et al. Molecules. 2013 18:12051 [PMID: 24084014].

[10] Taetz S et al. Pharm Res. 2006 23:1031 1037.[PMID: 16715394].

[11] Fedoroff OY et al. Biochemistry. 1998 37:12367 [PMID: 9730808].

[12] Gavathiotis E et al. J Mol Biol. 2003 334:25 [PMID: 14596797]. 
[13] Kim MY et al. J Am Chem Soc. 2002 124:2098 [PMID: 11878947]

[14] Mita H et al. Biochemistry. 2006 45:6765 [PMID: 16734413].

[15] Sun H et al. Bioorg Med Chem Lett. 2006 16:3586 [PMID: 16621536].

[16] Zhang WJ et al. Bioorg Med Chem. 2007 15:5493 [PMID: 17574421].

[17] Brassart B et al. Mol Pharmacol. 2007 72:631 [PMID: 17586599].

[18] Li J et al. Bioorg Med Chem Lett. 2011 21:6969 [PMID: 22018460].
[19] Li Q et al. Biochimie. 2009 91:811 [PMID: 19318115].

[20] Kubota T et al. Helv Chim Acta. 1982 65:611.

[21] Chen S et al. Oncotarget. 2017 8:10071 [PMID: 28052025].

[22] Radwa FF et al. J Clin Cell Immunol. 2011 S3:4 [PMID: 23336088].

[23] Lee S et al. Planta Med. 1998 64:303 [PMID: 9619109].

[24] Jiang J et al. Int J Mol Med. 2008 21:577 [PMID: 18425349].

[25] Yao X et al. Planta Med. 2012 78:1740 [PMID: 22961117].

[26] Gavathiotis E \& Searle MS, Org Biomol Chem. 2003 1:1650 [PMID: 12926351].

Edited by $\mathbf{P}$ Kangueane

Citation: Sillapapongwarakorn et al. Bioinformation 13(9): 284-292 (2017) License statement: This is an Open Access article which permits unrestricted use, distribution, and reproduction in any medium, provided the original work is properly credited. This is distributed under the terms of the Creative Commons Attribution License 